\title{
Biodegradation kinetics of linear alkylbenzene sulphonates in sea water
}

\author{
Jose A. Perales*, Manuel A. Manzano, M. Carmen Garrido, Diego Sales \& Jose M. Quiroga \\ Area of Environmental Technologies, Faculty of Marine and Environmental Sciences, Campus of Puerto Real. \\ Polígono Río San Pedro s/n, 11500, Puerto Real, Cádiz, Spain (*author for correspondence: e-mail: \\ joseantonio.perales@uca.es)
}

Accepted 13 December 2005

Key words: biodegradation, kinetic, linear alkylbenzene, sea water, surfactants, xenobiotics

\begin{abstract}
This article reports the primary biodegradation kinetics of linear alkylbenzene sulphonates (LAS) in sea water from the Bay of Cadiz (South West of the Iberian Peninsula). The authors used the biodegradation test guideline proposed by the Office of Prevention, Pesticides, and Toxic Substances of the United States Environmental Protection Agency; 835.3160 "Biodegradability in sea water" in its shake flask variant. High performance liquid chromatography (HPLC) has been employed for the analysis of the surfactant material. The surfactant shows a primary biodegradation kinetic in accordance with a logistic model, the kinetic parameters $t_{50}$ and lag time were calculated by means of a easy quantitative procedure introduced. Mean values of $6.15 \pm 0.45$ and $6.67 \pm 0.6$ days were obtained for $t_{50}$ and lag time, respectively. These results indicate that although LAS has a high primary biodegradation rate in sea water, it biodegrades slower than in similar tests conducted in river water.
\end{abstract}

\section{Introduction}

An ecological risk assessment is a process that evaluates the likelihood that adverse ecological effects may occur or are occurring as a result of exposure to one or more stressors. In Europe, risk assessment legislation included the Commission Directive 93/67/EEC (EC 1993a) which covers risk assessment of new substances while Commission Regulation No. 1488/94 (EC 1994), required under Council Regulation 793/93 (EC 1993b), lays down the risk assessment principles for existing substances.

One of the steps of an environmental risk assessment is the calculation of the predicted environmental concentrations. It is necessary to address short-term exposures, such as an accidental spill, which may result in high concentrations in an ecosystem but for a relatively short period of time, and long-term exposures that are the result of continuous discharges of used products by the consumer. An important part of the exposure assessment is to understand the biodegradation profile of the chemical and the extent to which we expect it to be removed from the environment considered.

Although in tonnage soap is still the most widely used surface agent on a world scale, the great variety of processes in which these types of substance are involved has led to a spectacular increase in usage, with consumption growing from approximately 13 million tonnes in 1977 to 18 million tonnes in 1995 (Granados 1996). Of this latest total, somewhat more than $50 \%\left(9.5 \times 10^{6}\right.$ tonnes/year $)$ correspond to soap and the rest $\left(8.5 \times 10^{6}\right.$ tonnes/year $)$ to synthetic surfactants (Granados 1996), of which Linear Alkylbenzene Sulphonates (LAS), with a consumption volume in 1995 of $1.5 \times 10^{6}$ tonnes, constitute $18 \%$ of the total of synthetic surfactants and $58.5 \%$ of the five leading types of synthetic surfactant, by consumption (Granados 1996).

LAS constitute one of the best-studied groups of chemicals in the aquatic environment to date (Belanger et al. 2002; OECD 1995; Painter \& 
Zabel 1988; Temmink \& Klapwijk 2004). Despite the numerous studies about LAS biodegradability in continental waters, there have been relatively few studies of LAS biodegradability in marine media. This is a deficiency since the sea is one of the main sumps for domestic wastewater, the main source of LAS to the marine environment.

This study presents the results of applying the biodegradation test proposed by the USEPA (United States Environmental Protection Agency) "Biodegradability in sea water" OPPTS (Office of Prevention, Pesticides, and Toxic Substances) 835.3160 (USEPA 1998) in its shake flask variant, to the surfactant sodium linear alkylbenzene sulphonate. The main objectives of this work has been: First, to test the biodegradability of this xenobiotic surfactant in a medium whose characteristics (low concentration of biomass, nutrients, etc.) could present a reduced self-purifying capacity, in comparison with other natural aquatic ecosystems. The second is to introduce an easy and more rigorous procedure than the graphical one to calculate the kinetic parameters of the biodegradation process. And finally to study the repeatability of the method proposed by the USEPA. Given that it deals with a test in which natural waters are employed, its possible variability is an aspect of great interest.

\section{Materials and methods}

All the biodegradation tests conducted in the present study follow the guideline OPPTS 835.3160 "Biodegradability in sea water" (USEPA 1998). This guideline covers two possible test methods, that of the closed bottle and that of the shake flask; the latter method has been employed in the present study. This method is a variant for sea water of the modified test guideline of the OECD (OECD 1992) that was developed by the Danish Institute of Water Quality for the European Community as a result of an exercise of intercalibration (Nyholm \& Kristensen 1987). A positive result in the test may be concluded that there is a potential for biodegradation in the marine environment. However, a negative result does not preclude such potential but indicates that further study is necessary, for example using as low a concentration of the test substance as possible.
Biodegradation test have been conducted on two compounds. The first is sodium benzoate, which is usually utilized as reference substrate in this type of test (USEPA 1998), since it is not considered to present problems of biodegradability, toxicity, volatility or low solubility. The second is the surfactant linear alkylbenzene sulphonate sodium salt (LAS), which presents an acceptable solubility in water but, given its amphiphilic character, could be susceptible to phenomena of adsorption onto the walls of the reactor vessels in which the biodegradation tests are performed. For this reason, it was decided to perform some abiotic tests to check the extent to which this factor might participate, together with the biodegradation, in the removal of the chemical from the test medium.

The following are the characteristics and properties of the compounds utilized:

Linear alkylbenzene sulphonate (LAS):

Supplier: Fluka Chemie A.G.; Product: Dodecylbenzenesulphonic acid sodium salt; Product No.: 44200; Empirical formula: $\mathrm{C}_{18} \mathrm{H}_{29} \mathrm{NaSO}_{3}$; Molecular mass: 348.48 g/mol; Aspect: Pale yellow powder; Solubility in water $\left(20^{\circ} \mathrm{C}\right): 50 \mathrm{~g} / 1$; Purity: $80.2 \%$; Content in carbon: $48.17 \%$; Composition: $80.2 \%$ of a mixture of homologues $\left(\mathrm{C}_{10}-\mathrm{C}_{13}\right)$, $\approx 17 \%$ Sodium sulphate, $<3 \%$ Water

Sodium benzoate:

Supplier: Fluka Chemie A.G.; Product: Sodium Benzoate purum p. a.; Product No.: 71300; Empirical formula: $\mathrm{C}_{7} \mathrm{H}_{5} \mathrm{O}_{2} \mathrm{Na}$; Molecular mass: $144.11 \mathrm{~g} / \mathrm{mol}$; Aspect: Crystalline white solid; Solubility in water $\left(20{ }^{\circ} \mathrm{C}\right): 660 \mathrm{~g} / \mathrm{l}$; Purity: $\geq 99 \%$; Carbon content: $58.29 \%$.

The source of the sea water utilized was a point in the Bay of Cadiz (South West of the Iberian Peninsula) (Figure 1). The sea water samples were taken with a Ruttner oceanographic type bottle at a depth of $0.5 \mathrm{~m}$; they were stored in polyethylene containers of 251 capacity and were transported to the laboratory immediately after sampling.

Once in the laboratory, the samples of sea water were filtered to remove coarse particles; for this, filters of fiberglass of $47 \mathrm{~mm}$ diameter and $1 \mu \mathrm{m}$ nominal pore size (Micron Separations Inc.) were employed. The general water quality characteristics of the natural test water used in shake flask batch tests are presented in Table 1 .

The vessels employed in the biodegradation tests had a capacity of 2.51 , were of borosilicate glass, and amber in color to ensure that the 


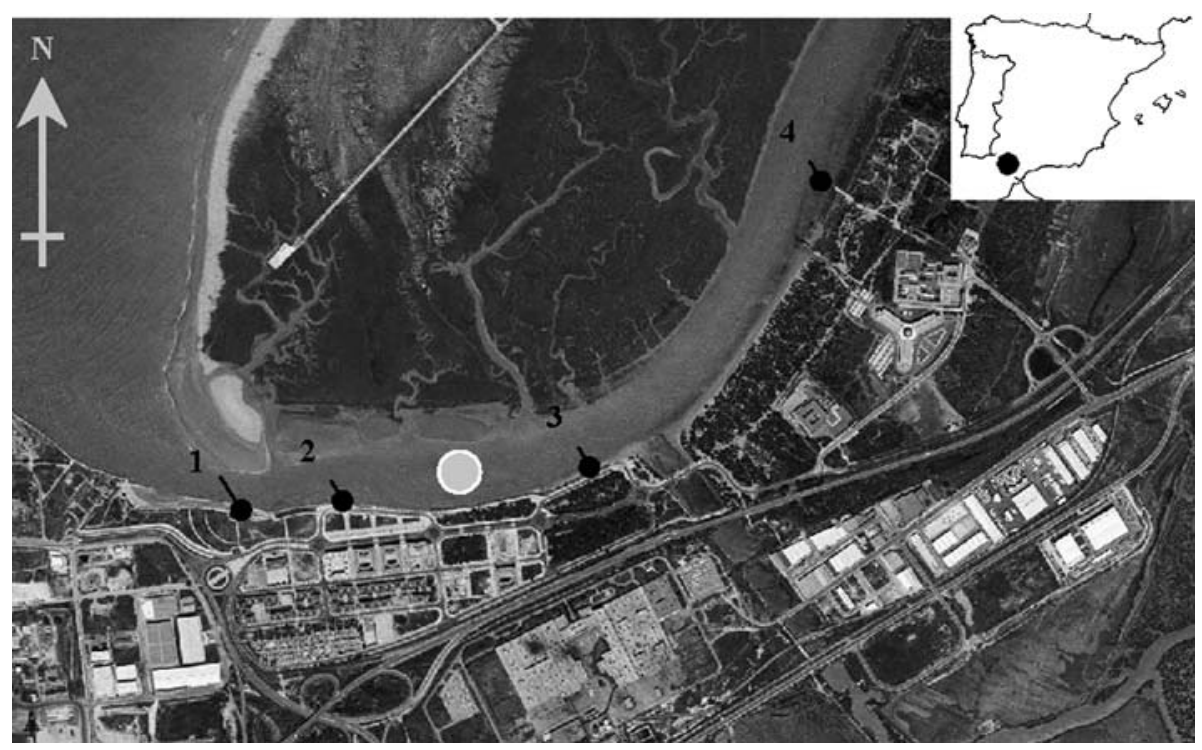

Figure 1. Image of the zone selected for taking samples of the sea water for the biodegradation tests. Points 1, 2 and 3 indicate the location of three urban storm-water discharges and point 4 is a domestic wastewater discharge $\left(<1000 \mathrm{~m}^{3} / \mathrm{d}\right)$.

biodegradation took place under diffuse light. The reactors were stoppered with a Polytetrafluoroethylene (PTFE) plug with holes that permitted the exchange of gases with the atmosphere. A volume of

Table 1. Analysis of sea water used in the biodegradation test $(n=3, \alpha=0.05)$

\begin{tabular}{|c|c|c|c|}
\hline Parameter & Units & Mean & $\begin{array}{l}\text { Standard } \\
\text { deviation }\end{array}$ \\
\hline $\mathrm{pH}$ & - & 8.11 & - \\
\hline Salinity & - & 36 & - \\
\hline Suspended solids & $\mathrm{mg} / \mathrm{l}$ & 224.0 & 56.0 \\
\hline Volatile solids & $\mathrm{mg} / 1$ & 31.5 & 6.4 \\
\hline$\%$ Volatile solids & - & 14.2 & 0.8 \\
\hline Total carbon & $\mathrm{mg} / \mathrm{l}$ & 29.76 & - \\
\hline Inorganic carbon & $\mathrm{mg} / \mathrm{l}$ & 24.72 & - \\
\hline Organic carbon & $\mathrm{mg} / \mathrm{l}$ & 5.04 & - \\
\hline Nitrites & $\mu \mathrm{g} / 1 \mathrm{NO}_{2}^{-}$ & 28.6 & 3.1 \\
\hline Nitrates & $\mu \mathrm{g} / 1 \mathrm{NO}_{3}^{-}$ & 20.6 & 0.9 \\
\hline Ammonium & $\mu \mathrm{g} / 1 \mathrm{NH}_{4}^{+}$ & 20.3 & 2.1 \\
\hline Total phosphorus & $\mu \mathrm{g} / 1 \mathrm{P}$ & 114.9 & 4.2 \\
\hline Silicates & $\mu \mathrm{g} / 1 \mathrm{Si}$ & 257.4 & 25.5 \\
\hline Greases and oils & $\mathrm{mg} / 1$ & $<2$ & - \\
\hline Anionic surfactants & $\mu \mathrm{g} / 1 \mathrm{MBAS}$ & $<40$ & - \\
\hline Phenols & $\mu \mathrm{g} / 1 \mathrm{C}_{6} \mathrm{H}_{5} \mathrm{OH}$ & $<25$ & - \\
\hline \multirow[t]{3}{*}{ Chlorophyll } & $\mu \mathrm{g} / \mathrm{l} \mathrm{Chl} a$ & 105 & - \\
\hline & $\mu \mathrm{g} / \mathrm{l} \mathrm{Chl} b$ & n.d. & - \\
\hline & $\mu \mathrm{g} / \mathrm{l} \mathrm{Chl} c$ & n.d. & - \\
\hline Fecal streptococci & $\mathrm{UFC} / 100 \mathrm{ml}$ & 6700 & - \\
\hline
\end{tabular}

1.51 of filtered sea water was added to each reactor with the objective of leaving sufficient empty space to allow the correct reoxygenation of the medium.

Next, a volume of $1.5 \mathrm{ml}$ of each of the four stock solutions of mineral nutrients (USEPA 1998) was added. Having been agitated for $15 \mathrm{~min}$, the reactors were stoppered and kept in a precision incubator (Hot-Cold GL 4000700: J. P. Selecta S. A.) at $20 \pm 1{ }^{\circ} \mathrm{C}$ and in darkness for $24 \mathrm{~h}$ before the compounds to be tested were added; the purpose of this was to allow the preconditioning of the microorganisms to the test conditions.

The experiment conducted consisted of a biodegradation test of the linear alkylbenzene sulphonate sodium salt, in quintuplicate, and with an initial concentration of surfactant material close to $20 \mathrm{mg} / 1$ (T1, T2, T3, T4 and T5). In parallel, an abiotic biodegradation test was conducted with the same initial surfactant concentration, but including mercury chloride at a concentration of $100 \mathrm{mg} /$ 1 (ABI); at the same time a reference test was conducted with an easily biodegradable substrate, sodium benzoate, at an initial concentration of approximately $20 \mathrm{mg}$ Dissolved Organic Carbon (DOC)/1 (REF), in order to check the activity of the microbial population present in the test medium. Lastly, a control test, in duplicate, was included, that only contained the test medium and the nutrients (B1 and B2). 
The determination of the LAS was by means of high performance liquid chromatography (HPLC) in reverse phase, following the method proposed by Nakae et al. (1980). The equipment employed was a chromatograph consisting of two pumps, one a Waters model 510 alternating double piston type and the other a Waters model 501 simple piston type. The injection unit was a manual type model U6K, and the detection system was of the fluorescence type, Waters model 470. In the case of the reference test (REF), the determination of the content of substrate (sodium benzoate) was by means of the analysis of the concentration of DOC using a Shimadzu total organic carbon 5050A analyser.

The values of concentration of LAS and/or DOC obtained throughout the experiment, for all the tests conducted, enabled the course of the biodegradation to be monitored over the test period. For the treatment of the results, the USEPA directive proposes the graphical calculation of the values of the lag time $\left(t_{\mathrm{L}}\right)$ and time starting from the end of the lag phase to reach $50 \%$ of biodegradation $\left(t_{50}\right)$. However, to be more rigorous, it was decided to utilize another procedure consisting of fitting the experimental data obtained in the various tests to the different biodegradation kinetic models proposed by Simkins \& Alexander (1984). Then having selected the simplest model that best fitted the experimental results, the values of $t_{\mathrm{L}}$ and $t_{50}$ were calculated in accordance with the definitions proposed in the guideline (USEPA 1998). The computational process used to fit the data to the models was a non-linear estimation (user-specified regression), of the quasi-Newton method. The estimation conditions were: convergence criterion, 0.001; maximum number of iterations, 100; start values for all the parameters, 0.1 and initial step, 0.001 . The software used was Statistica 4.0 (Statsoft, Inc. 1993).

\section{Results and discussion}

Figure 2 shows the evolution followed by the concentration of substrate in the abiotic test (ABI) and in the reference test (REF).

It is observed that the values of concentration of LAS in the medium of the abiotic test remained around $20 \mathrm{mg} / 1$ throughout the period

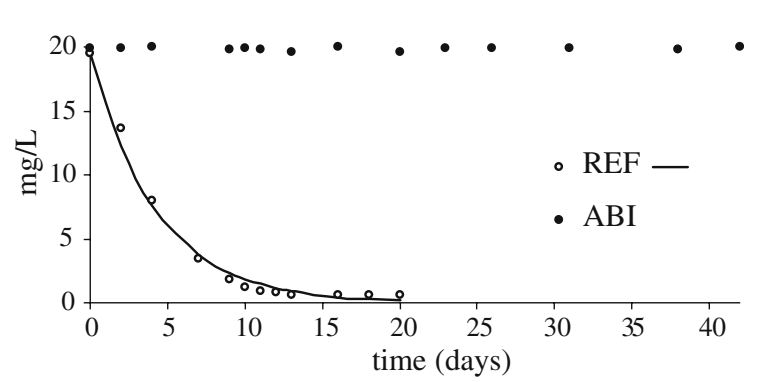

Figure 2. Evolution of the substrate present in the medium, in the abiotic (ABI, mg LAS/l) and reference (REF, mg DOC/1) tests.

of biodegradation. Specifically, the percentage of the mean residual over the course of the test was $100.2 \%$, with a standard deviation of 0.7 . From this result the contribution of abiotic processes to the elimination of LAS can be discounted in the biodegradation tests conducted.

In respect of the reference test (REF), it can be observed from Figure 2 that the sodium benzoate at an initial concentration of $19.5 \mathrm{mg} / \mathrm{l}$ of DOC was biodegraded to an extent of more than $97 \%$, after 13 test days.

As already stated, a kinetic modeling of the experimental results obtained was performed. For the case of the reference test, Table 2 summarizes the results obtained for those models that showed strong correlations $\left(r^{2}>0.99\right)$ with experimental data.

As can be observed in Table 2, the parameter $\mathrm{B}_{0}$ of the logistic model (concentration of substrate required to produce the initial concentration of microorganisms) presents a no consistent high value, given that the test medium in all cases was natural sea water and therefore had relatively low concentrations of microorganisms. In respect of the model of Quiroga, Sales \& Romero (Quiroga et al. 1999), the parameter $S_{t_{0}}$ should be slightly higher than the initial concentration of substrate employed in the test; this did not occur, thus robbing the value obtained of sense. From the arguments presented so far, it can be concluded that the kinetic model that best represents the behavior observed in the reference test of biodegradation is the first order model (Figure 2, unbroken line).

If the lag time $\left(t_{\mathrm{L}}\right)$ is taken as the period elapsed until $10 \%$ of biodegradation is reached (USEPA 1998), to calculate its value it is sufficient to substitute, in the kinetic expression corresponding to 
Table 2. Kinetic parameters corresponding to the reference test

\begin{tabular}{|c|c|c|c|}
\hline Model & Expression & & \\
\hline First order (Simkins \& Alexander 1984) & $S=s_{0} \cdot \mathrm{e}^{-K_{1} \cdot t}$ & $K_{1}=\mu_{\max } \cdot\left(\frac{B_{0}}{K_{\mathrm{s}}}\right)\left(\mathrm{d}^{-1}\right)$ & $\begin{array}{l}0.235 \\
0.991\end{array}$ \\
\hline Logistic (Simkins \& Alexander 1984) & $S=\frac{\left(S_{0}+B_{0}\right)}{1+\left(\frac{B_{0}}{S_{0}}\right) \cdot e^{\left[K_{\mathrm{Lg}} \cdot\left(S_{0}+B_{0}\right) \cdot t\right]}}$ & $\begin{array}{l}B_{0}(\mathrm{mg} / \mathrm{l}) \\
K_{\mathrm{Lg}}=\frac{\mu_{\max }}{K_{\mathrm{S}}} \\
(\mathrm{mg} / \mathrm{l} / \mathrm{d})\end{array}$ & $\begin{array}{r}17.550 \\
0.009\end{array}$ \\
\hline Quiroga-Sales-Romero (Quiroga et al. 1999) & $S=\frac{S_{t_{0}} \cdot\left(S_{0}-S_{\mathrm{nb}}\right)+S_{\mathrm{nb}} \cdot\left(S_{0}-S_{t_{0}}\right) \cdot e^{\mu_{\max } \cdot t}}{\left(S_{0}-S_{\mathrm{nb}}\right)+\left(S_{0}-S_{t_{0}}\right) \cdot e^{\mu_{\max } \cdot t}}$ & $\begin{array}{l}r^{2} \\
S_{t_{0}} \mathrm{mg} / \mathrm{l} \\
S_{\mathrm{nb}}(\mathrm{mg} / \mathrm{l}) \\
\mu_{\max }\left(\mathrm{d}^{-1}\right) \\
r^{2}\end{array}$ & $\begin{array}{l}0.998 \\
-10^{7} \\
-0.264 \\
0.226 \\
0.992\end{array}$ \\
\hline$t$ & \multicolumn{3}{|l|}{ time $(\mathrm{d})$} \\
\hline$S_{0}$ & \multicolumn{3}{|l|}{ initial concentration of substrate $(\mathrm{mg} / \mathrm{l})$} \\
\hline$S$ & \multicolumn{3}{|l|}{ concentration of substrate at time $t(\mathrm{mg} / \mathrm{l})$} \\
\hline$B_{0}$ & \multicolumn{3}{|c|}{ substrate required to produce the initial microbial concentration $(\mathrm{mg} / \mathrm{l})$} \\
\hline$\mu_{\max }$ & \multicolumn{3}{|c|}{ maximum specific rate of growth of microorganisms $\left(\mathrm{d}^{-1}\right)$} \\
\hline$K_{\mathrm{s}}$ & \multicolumn{3}{|c|}{$\begin{array}{l}\text { concentration of substrate at which the specific rate of growth of microorganisms is } \\
\text { equal to half of the maximum }\left(\mu_{\max } / 2\right) .(\mathrm{mg} / \mathrm{l})\end{array}$} \\
\hline$S_{t_{0}}$ & \multicolumn{3}{|c|}{ maximum concentration of substrate convertible into biomass $\left(B_{0}+S_{0}\right)(\mathrm{mg} / \mathrm{l})$} \\
\hline$S_{\mathrm{nb}}$ & \multicolumn{3}{|c|}{ concentration of non-biodegradable substrate $(\mathrm{mg} / \mathrm{l})$} \\
\hline
\end{tabular}

the test REF (Equation (1)), the term $S$ for $0.9 \times S_{0}$, and this expression can be obtained:

$$
t_{\mathrm{L}}=t_{10}=-\frac{\operatorname{Ln}(0.9)}{K_{1}}
$$

Further, considering $t_{50}$ as the period elapsed from the end of the acclimatization period until $50 \%$ of biodegradation is reached (USEPA 1998), this is given by the expression:

$$
t_{50}=t_{1 / 2}-t_{10}
$$

From which one obtains:

$$
t_{50}=\frac{\operatorname{Ln}(0.9 / 0.5)}{K_{1}}
$$

Substituting the value of the first order kinetic constant $\left(K_{1}\right)$ by that obtained for the reference test (REF), $0.235 \mathrm{~d}^{-1}$ (Table 2), values for the lag time of 0.45 and 2.50 days for $t_{50}$ are obtained. These values can be considered consistent with the results that Nyholm \& Kristensen (1987) reports of 1-4 days for the lag period and of 1-7 days for $t_{50}$. The results can be taken to indicate that the microbial population presents an activity within the normal range and therefore will not limit the biodegradation rate in the tests with LAS.

In Figure 3 are given the values of the concentration of LAS presented in the test medium at various stages of the process of biodegradation, for the five experiments conducted with $20 \mathrm{mg} / 1$ of LAS.

First, it can be observed how, in all the tests, the removal of more than $90 \%$ of LAS was achieved within about 20 days. It can also be seen that the biodegradation curves obtained for the five replicate tests appear fairly similar one to another so it seems that the biodegradation test shows a good repeatability. However, this initial qualitative evaluation must be quantified. This involves determining the rate of biodegradation obtained in each replicate, i.e. the values of the lag period and $t_{50}$. As was done in the case of the reference test, the kinetics of biodegradation of the five tests had been modeled; the models presenting the best fit to the experimental data are given in Table 3.

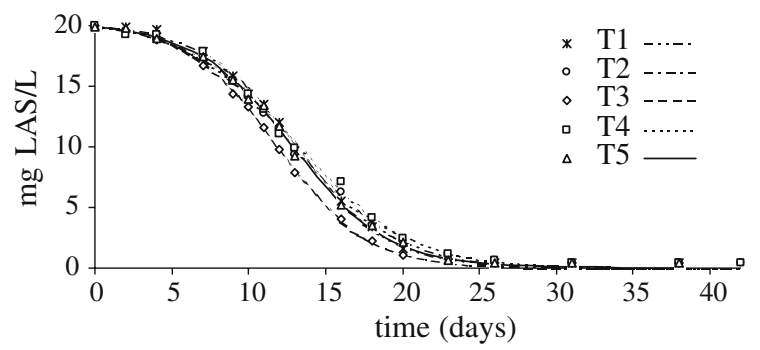

Figure 3. Evolution of LAS concentration in the biodegradation tests. 
Table 3. Kinetic parameters corresponding to the LAS biodegradation tests

\begin{tabular}{llcrrrr}
\hline & & T1 & T2 & T3 & T4 & T5 \\
\hline Logistic (Simkins \& Alexander 1984) & $B_{0}(\mathrm{mg} / \mathrm{l})$ & 0.267 & 0.473 & 0.421 & 0.565 & 0.332 \\
& $K_{\mathrm{Lg}}(\mathrm{mg} / \mathrm{l} / \mathrm{d})$ & 0.016 & 0.014 & 0.016 & 0.013 & 0.016 \\
& $r^{2}$ & 0.999 & 0.999 & 0.999 & 0.997 & 0.998 \\
Quiroga-Sales-Romero (Quiroga et al. 1999) & $S_{t_{0}} \mathrm{mg} / \mathrm{l}$ & 20.19 & 20.295 & 20.365 & 20.437 & 20.143 \\
& $S_{\mathrm{nb}}(\mathrm{mg} / \mathrm{l})$ & 0.256 & 0.325 & 0.258 & 0.289 & 0.294 \\
& $\mu_{\max }\left(\mathrm{d}^{-1}\right)$ & 0.342 & 0.303 & 0.341 & 0.286 & 0.331 \\
& $r^{2}$ & 0.999 & 0.999 & 0.999 & 0.998 & 0.998 \\
\hline
\end{tabular}

In Table 3 it is observed that in this case there are two kinetic models that shows strong correlations with the experimental results. Further, neither of the two models presented values of the kinetic parameters that would allow one or other to be discounted, as happened in the reference test; therefore the better model must be selected on the criterion of simplicity. Since in this case the logistic model presents fewer kinetic parameters (Equation (2)), this is the model that had been selected as representative of the process of biodegradation in the five tests. In Figure 3 the values that this model predicted are represented by lines; it can be confirmed that the experimental values fitted fairly well to those predicted by the logistic kinetic model.

Following the same criterion as that adopted in the preceding case with the reference test, working out the value of time from expression 2 gives:

$$
t=\frac{\operatorname{Ln}\left[\left(\frac{S_{0}}{B_{0}}\right) \cdot\left(\frac{\left(S_{0}+B_{0}\right)}{S}-1\right)\right]}{K_{\mathrm{Lg}} \cdot\left(S_{0}+B_{0}\right)}
$$

To calculate the value of the period of acclimatization, it is sufficient to substitute the term $S$ by $0.9 \times S_{0}$, whereas to determine the $t_{50}$ it is sufficient to subtract the lag time $\left(t_{\mathrm{L}}=t_{10}\right)$ from the half life or time required to reach $50 \%$ of biodegradation $\left(t_{1 / 2}, S=0.5 \times S_{0}\right)$.

$$
\begin{array}{r}
t_{\mathrm{L}}=\frac{\operatorname{Ln}\left[\frac{1}{9} \cdot\left(\frac{S_{0}}{B_{0}}+10\right)\right]}{K_{\mathrm{Lg}} \cdot\left(S_{0}+B_{0}\right)} \\
t_{50}=t_{1 / 2}-t_{\mathrm{L}}=\frac{\operatorname{Ln}\left[\cdot\left(\frac{9 \cdot\left(S_{0}+2 \cdot B_{0}\right)}{\left(S_{0}+10 \cdot B_{0}\right)}\right)\right]}{K_{\mathrm{Lg}} \cdot\left(S_{0}+B_{0}\right)}
\end{array}
$$

From these two expressions (8 and 9), the lag times and those of $t_{50}$ had been calculated for the different biodegradation tests conducted. The values obtained are shown represented in Figure 4.

The close similarity between the replicate tests conducted continues to be observed; mean $t_{50}$ and lag times of 6.15 and 6.67 days, respectively, with standard deviations of 0.44 and 0.48 days, respectively, are presented; this means that the $95 \%$ confidence limits are given by:

$$
\begin{aligned}
& t_{\mathrm{L}}=6.15 \pm 0.54 \text { days } \\
& t_{50}=6.67 \pm 0.60 \text { days }
\end{aligned}
$$

An usual form of presenting the variability of the results is by means of the relative standard deviation, also known as the coefficient of variation; this is given by the following expression:

$$
\mathrm{CV}=100 \cdot \frac{s}{\bar{x}}
$$

where:

$s=$ standard deviation

$\bar{x}=$ mean value

In accordance with this expression and the results obtained, the resulting coefficients of variation for the times of $t_{50}$ and lag are $7.2 \%$ and $7.1 \%$, respectively.

In a previous work (Perales et al. 1999) the authors study the primary biodegradation kinetics of LAS in river water. In the test conducted at $21{ }^{\circ} \mathrm{C}$ with $20 \mathrm{mg} \mathrm{LAS} / 1$, the lag time and $t_{50}$ obtained (according with the previous definition) were 3.26 and 2.03 days, respectively. Approximately two and three times lower, than the lag time and $t_{50}$ presented in this work, respectively.

If we compare the test conditions for both experiments:

- Similar temperature and initial surfactant concentration

- The environmental quality of the test medium was pristine water in the case of the tests 


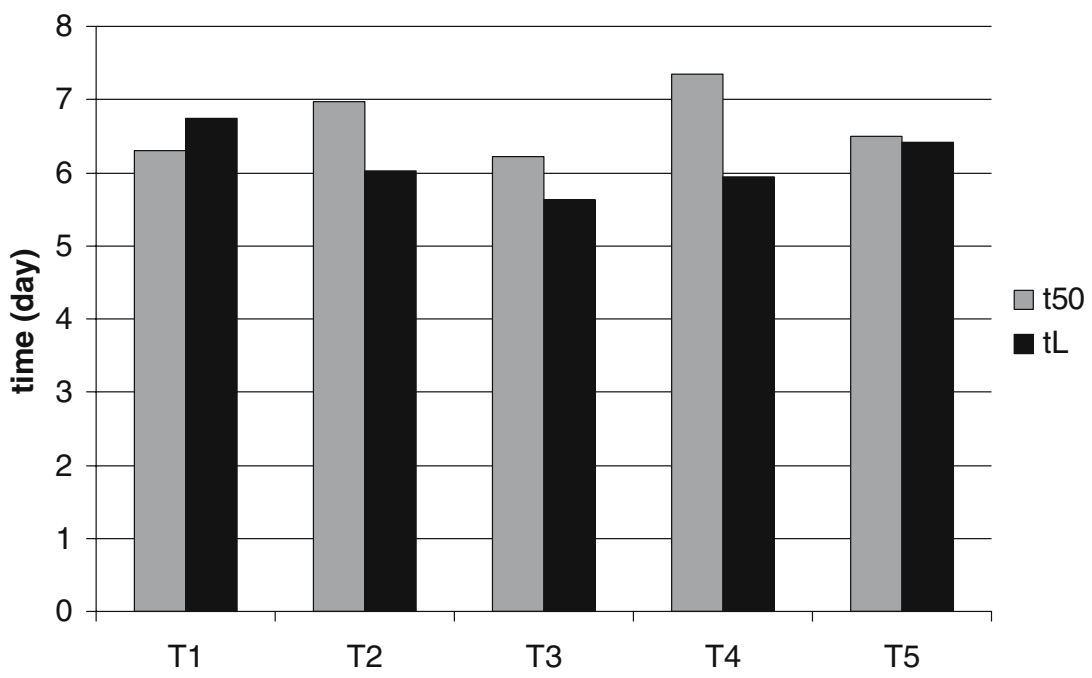

Figure 4. Time of acclimatization $\left(t_{\mathrm{L}}\right)$ and $t_{50}$ in the five LAS biodegradation tests.

conducted with river water and slightly anthropizated coastal water in the sea water biodegradation tests. (Table 2)

- Mixture of LAS with an average alkyl chain of 11.5 and 11.13 atoms of carbon in the tests conducted with sea and river water, respectively

- In the river water test no nutrient addition was included

It is possible to consider, that under the test condition used, the biodegradation kinetic of LAS in river water is faster than in sea water. This lower biodegradability potential of marine aquatic environments has been observed by other authors for other chemicals like Bisphenol A (Jeong-Hun \& Fusao 2005); benzo(a)pyrene (Kot-Wasik et al. 2004) and poly(3-hydroxybutyrate) homopolymer, P(3HB) (Kasuya et al. 1998).

\section{Conclusions}

The following conclusions can be drawn from the biodegradation tests carried out in this work:

1. LAS are surfactants that present a high rate of primary biodegradation in sea water, having $t_{50}$ comparable to those presented by the mineralization of compounds considered to be reference substances.

2. LAS, like other chemicals, biodegrades slower in sea water than in river water. This fact together with the lack of studies related with the fate of chemicals in marine environments compared with other media (wastewaters or lake/river waters) makes necessary to deep in testing of the biodegradation in sea water of this and other chemicals in order to have adequate data to carry out precise environmental risk assessments in marine environments.

3. The methodology proposed for the treatment of the data from the biodegradation test can be considered as a scientifically more rigorous alternative for the calculation of the rates of biodegradation than that the graphical one proposed in the USEPA directive, and in addition provides much more information relating to the biodegradation kinetic of the chemical.

4. The low coefficients of variation for the kinetic parameters obtained indicated that the biodegradation test method in sea water proposed by the USEPA in its shake flask variant shows a good repeatability with the chemical tested. Nevertheless its reproducibility should be studied using waters from different locations, in order to determine the variability of the biodegradation kinetics of LAS in sea water.

\section{References}

Belanger SE, Bowling JW, Lee DM, LeBlanc EM, Kerr KM, McAvoy DC, Christman SC \& Davidson DH (2002) Integration of aquatic fate and ecological responses to linear alkyl benzene sulphonate (LAS) in model stream ecosystems. Ecotoxicol. Environ. Saf. 52(2): 150-171 
EC (European Commission) (1993a) Commission Directive 93/67/EEC of 20 July 1993 laying down the principles for assessment of risks to man and the environment of substances notified in accordance with Council Directive 67/548/ EEC. Official J. L 227(08/09/1993): 9-18

EC (European Commission) (1993b) Council Regulation (EEC) No 793/93 of 23 March 1993 on the evaluation and control of the risks of existing substances. Official J. L 084(05/04/1993): $1-75$

EC (European Commission) (1994) Commission Regulation (EC) No 1488/94 of 28 June 1994 laying down the principles for the assessment of risks to man and the environment of existing substances in accordance with Council Regulation (EEC) No 793/93. Official J. L 161(29/06/1994): 3-11

Granados J (1996) Surfactant raw materials. Constant evolution and a solid future. 4th World Surf. Congr. 1: 100-123

Jeong-Hun K \& Fusao K (2005) Bisphenol A degradation in sea water is different from that in river water. Chemosphere 60(9): 1288-1292

Kasuya K, Takagi K, Ishiwatari S, Yoshida Y \& Doi Y (1998) Biodegradabilities of various aliphatic polyesters in natural waters. Polym. Degrad. Stab. 59(1-3): 327-332

Kot-Wasik A, Dbrowska D \& Namienik J (2004) Photodegradation and biodegradation study of benzo(a)pyrene in different liquid media. J. Photochem. Photobiol. A, Chem. 168(1-2): 109-115

Nakae A, Tsuji K \& Yamakana M (1980) Determination of trace amounts of alkylbenzenesulphonates by high performance liquid chromatography with fluorometric detection. Anal. Chem. 52(14): 2275-2277
Nyholm N \& Kristensen P (1987) Screening test methods for assessment of biodegradability of chemical substances in sea water. Final report of the ring test programme 1984-1985, March 1987, Commission of the European Communities

OECD (Organisation for Economic Co-operation and Development) (1992) OECD guidelines for the testing of chemicals. 301: Ready Biodegradability. OECD, Paris

OECD (Organisation for Economic Co-operation and Development) (1995) Environment monograph no. 98: Detailed Review Paper on Biodegradability Testing. OECD, Paris

Painter H \& Zabel T (1988) Review of the environmental safety of LAS. Technical Report CO 1659-M/1/EV 8658. Water Research Center. Medmenham, United Kingdom

Perales JA, Manzano MA, Sales D \& Quiroga JM (1999) Biodegradation kinetics of LAS in river water. Int. Biodeter. Biodegradation 43: 155-160

Quiroga JM, Perales JA, Romero LI \& Sales D (1999) Biodegradation kinetics of surfactants in sea water. Chemosphere 19(11): 1957-1969

Simkins S \& Alexander N (1984) Models for mineralisation kinetics with the variables of substrate concentration and population density. Appl. Environ. Microbiol. 47: 1299-1305

Temmink H \& Klapwijk B (2004) Fate of linear alkylbenzene sulphonate (LAS) in activated sludge plants. Water Res. 38(4): 903-912

USEPA (1998) Fate, transport and transformation test guidelines. Office of Prevention, Pesticides, and Toxic Substances. "Biodegradability in sea water" 835.3160. (http://www.epa.gov/ opptsfrs/OPPTS_Harmonized/835_Fate_Transport_and_Transformation_Test_Guidelines/Series/835-3160.pdf) 